\title{
Diagnostic Challenge of Sulcus Vocalis Made Easier
}

\author{
${ }^{1}$ Nupur Kapoor Nerurkar, ${ }^{2}$ Harsh Karan Gupta, ${ }^{3}$ Ajay Eknath Shedge
}

\begin{abstract}
Objectives: To introduce a simple diagnostic test performed with white light laryngoscopy for the diagnosis of sulcus vocalis.

Materials and methods: This is a retrospective observational study. A total of 14 patients with voice-related complaints and a phonatory gap on examination were included. Obvious structural and neuromuscular glottic pathologies were excluded. Phonatory gap was measured using white light rigid laryngoscopy with the technique described here. Findings were then correlated with stroboscopy.
\end{abstract}

Results: All 14 patients (10 U/L and $4 \mathrm{~B} / \mathrm{L}$ ), observed to have an asymmetric phonatory gap on white light rigid laryngoscopy, were diagnosed with sulcus vocalis.

Conclusion: An asymmetric phonatory gap, as seen on white light laryngoscopy and measured with the simple technique mentioned here, should make the laryngologist suspect a sulcus vocalis. However, the diagnosis needs to be confirmed by stroboscopy.

Keywords: Phonatory gap, Stroboscopy, Sulcus.

How to cite this article: Nerurkar NK, Gupta HK, Shedge AE. Diagnostic Challenge of Sulcus Vocalis Made Easier. Int J Phonosurg Laryngol 2015;5(2):39-41.

Source of support: Nil

Conflict of interest: None

\section{INTRODUCTION}

Sulcus vocalis has been defined as a groove or a depression on the vibratory surface of the vocal fold wherein the epithelium is tethered onto the subepithelial tissues, superficial lamina propria (SLP), or deeper. ${ }^{1}$ Ford et $\mathrm{al}^{2}$ have divided sulcus vocalis into three types depending upon the depth of migration of the epithelium. Type I, also known as physiological sulcus, is a longitudinal depression of the epithelium into the SLP but not down to the vocal ligament. Hence, the mucosal wave pattern is not altered and the patient is relatively asymptomatic. Type II (sulcus vergeture) is a full-length depression extending down to the vocal ligament or deeper. Type III,

\footnotetext{
${ }^{1}$ Consultant, ${ }^{2,3}$ Fellow

${ }^{1-3}$ Department of Otorhinolaryngology, Bombay Hospital Mumbai, Maharashtra, India
}

Corresponding Author: Harsh Karan Gupta, Fellow Department of Otorhinolaryngology, Bombay Hospital, Mumbai Maharashtra, India, Phone: 8652744412, e-mail: harsh_ karan21@hotmail.com on the contrary, is often deep pit-like, focal, and deeper indentation of epithelium with loss of deeper layers of lamina propria and often muscle (Fig. 1). This leads to loss of pliability and tissue volume; the otherwise smooth movement of the epithelial cover over the body is altered. ${ }^{2-6}$ The patients present with hoarseness and more often symptoms of glottal insufficiency like vocal fatigue, inability to talk loudly, effortful speech with odynophonia, and occasionally a high-pitched voice. ${ }^{7}$

Due to this depression within the vocal fold, a phonatory gap is commonly observed. This phonatory gap has been described as a 'spindle-shaped glottis'. Even though sulci are often bilateral, the depth often varies. In our experience, the phonatory gaps associated with sulci are more often asymmetrical or ' $\mathrm{D}$ ' shaped.

Most sulci remain undiagnosed because of lack of objective diagnostic criteria, clinician awareness, and occasionally subclinical symptoms. Our study aims to discuss a new diagnostic parameter, which also lends objectivity, to help the budding laryngologist pick-up a sulcus vocalis.

\section{MATERIALS AND METHODS}

This is a retrospective observational study of prospectively maintained database. In our voice clinic, 257 patients presented, from November 2014 to April 2015, with complaints of hoarseness and/or vocal fatigue and/or reduced range of speech. All these patients underwent white light rigid laryngoscopy and stroboscopy

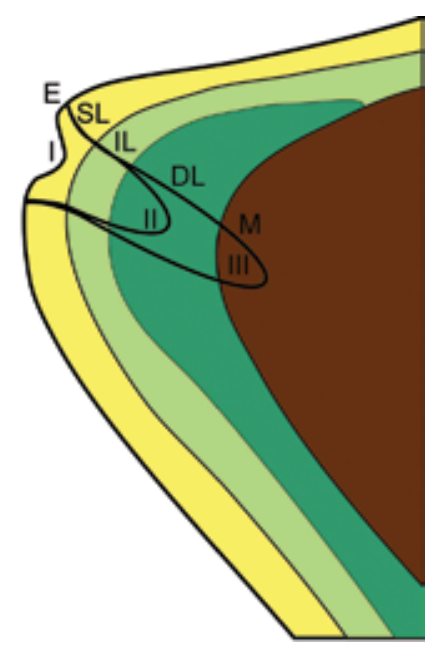

Fig. 1: Coronal section through the vocal fold depicting types I, II and III sulcus vocalis. (E: Stratified squamous epithelium, SL: Superficial lamina propria, IL: Intermediate lamina propria, DL: Deep lamina propria, M: Vocalis muscle) 
evaluation for an accurate anatomical and physiological assessment of the vocal folds.

Inclusion criterion was patients who had a phonatory gap on white light laryngoscopy examination.

Exclusion criteria were: (1) Any obvious glottic lesion like vocal fold cyst, polyp, nodule, papilloma, contact granuloma, leukoplakia, suspected vocal fold malignancy, (2) Patients with U/L or B/L vocal fold paralysis/paresis and (3) Patients with history of prior vocal fold surgery.

Still images of the recorded evaluations of white light rigid laryngoscopy with the vocal folds in the fully adducted position were studied (using Scopydoc version 7.0.2). Printouts were taken of these still images. A vertical line (L) was then drawn through the interarytenoid area (in adducted vocal folds) to the anterior commissure. The length of this line $\mathrm{L}$ was calculated and was divided into 10 equal parts or 'units.' The distance of the medial edges of both the vocal folds, at the point of maximum deviation, from the line $L$ was calculated in terms of the 'units' mentioned earlier. The distances were assigned values ' $\mathrm{Rd}^{\prime}$ and ' $\mathrm{Ld}$ ' for right and left vocal folds, respectively (Figs $2 \mathrm{~A}$ to $\mathrm{C}$ ). These findings were then correlated with the stroboscopy findings to evaluate the presence or absence of a sulcus vocalis. The association of a sulcus with $\mathrm{Rd}$ and $\mathrm{Ld}$ was analyzed.

\section{RESULTS}

Of the total 257 patients screened, 14 met the inclusion criterion and were assessed in detail. There were 12 males and 2 females. All 14 patients who had a phonatory gap on white light rigid laryngoscopy were diagnosed with sulcus vocalis on stroboscopy examination. The sulcus vocalis encountered were bilateral in 4 patients and unilateral in 10 patients (right-sided in 8 and left-sided in 2). Hence, a total of 18 vocal folds had sulcus vocalis.

On calculating the $\mathrm{Rd}$ and $\mathrm{Ld}$, it was seen that none of the 14 patients had a symmetric phonatory gap. The presence of a phonatory gap, i.e. Rd or Ld, correlated with the side of the sulcus vocalis (Figs 3 and 4). Also, greater the $\mathrm{Rd}$ or $\mathrm{Ld}$, deeper was the sulcus on that side, as confirmed on stroboscopy. The average phonatory gap of all the affected vocal folds (i.e. 18) was 0.485 units (Table 1).
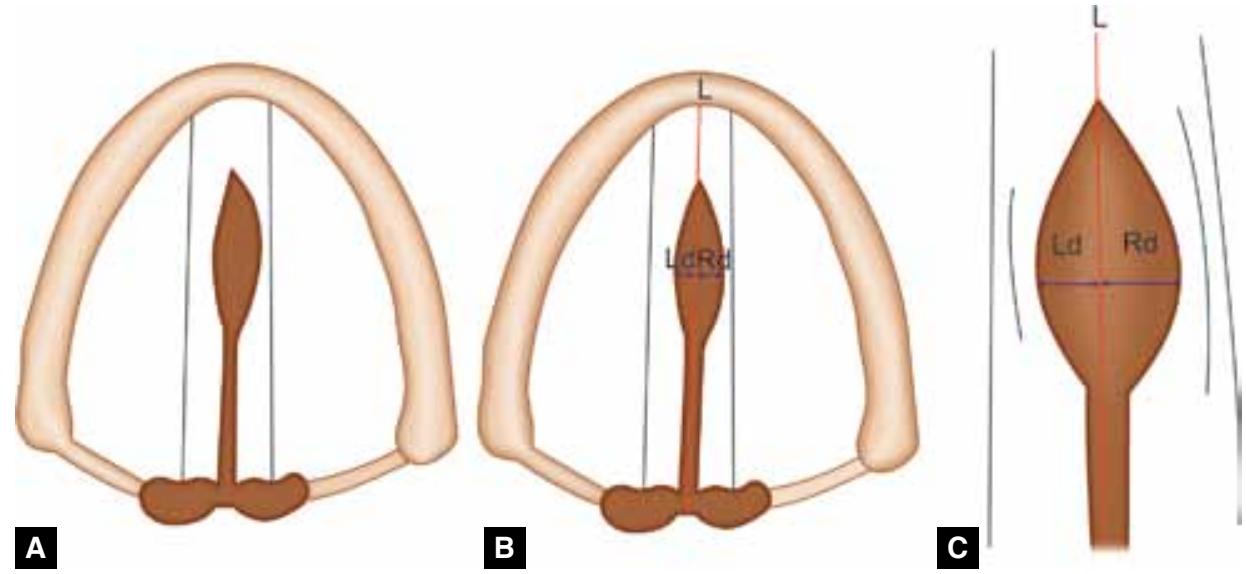

Figs 2A to C: Schematic illustration of calculation of phonatory gap: (A) Phonatory gap as seen on white light rigid laryngoscopy, (B) application of line L, Rd and Ld to measure the phonatory gap for each vocal fold and (C) magnified view of the phonatory gap

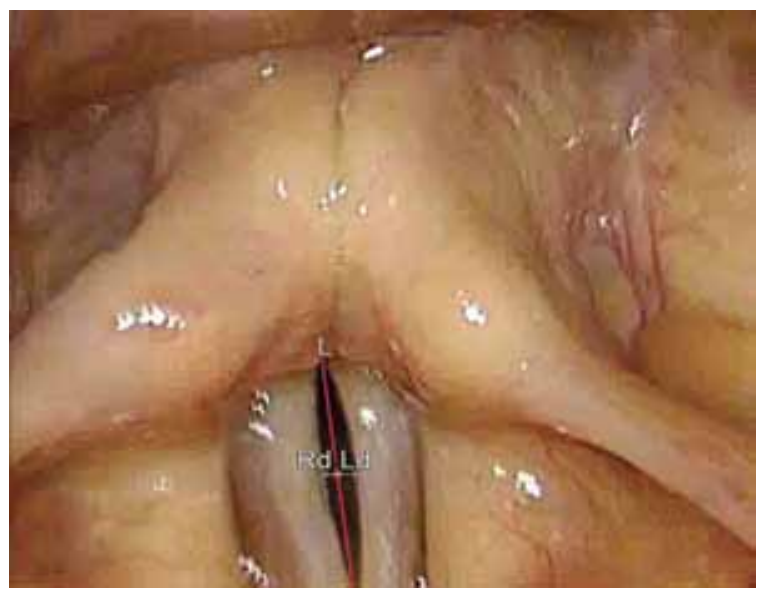

Fig. 3: Measuring phonatory gap in an image taken with vocal folds in maximum adducted position in a case of $B / L$ sulcus vocalis

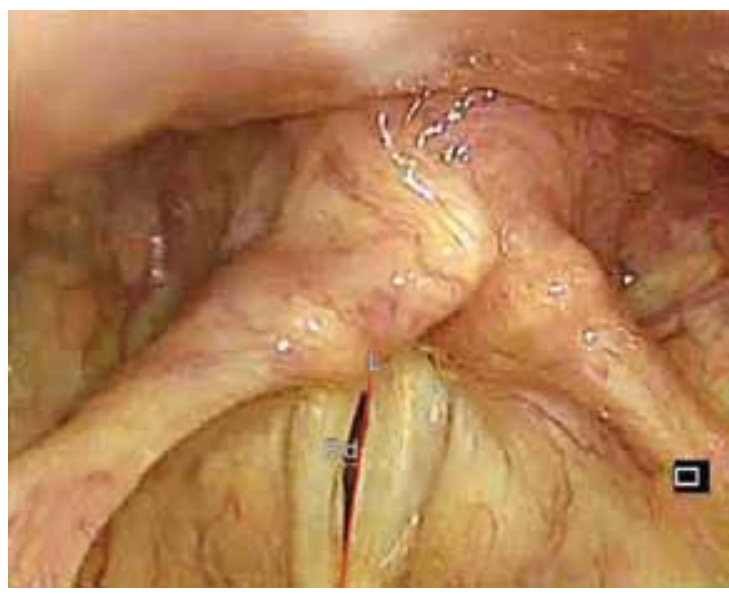

Fig. 4: Measuring phonatory gap in an image taken with vocal folds in maximum adducted position in a case of U/L (right sided) sulcus vocalis 
Table 1: Results

\begin{tabular}{rlll}
\hline SI. no. & $R d$ (units) & Ld (units) & Strobe findings \\
\hline 1. & 0.67 & - & Right-sided grade III sulcus \\
2. & 0.50 & - & Right-sided grade III sulcus \\
3. & 0.53 & 0.79 & B/L sulcus; left deeper than right \\
4. & 0.50 & 0.75 & B/L sulcus; left deeper than right \\
5. & 0.60 & 0.20 & B/L sulcus; right deeper than left \\
6. & - & 0.42 & Left-sided grade II sulcus \\
7. & 0.52 & - & Right-sided grade III sulcus \\
8. & 0.50 & - & Right-sided grade III sulcus \\
9. & 0.28 & - & Right-sided grade I sulcus \\
10. & 0.26 & - & Right-sided grade I sulcus \\
11. & 0.18 & 0.54 & B/L sulcus; left deeper than right \\
12. & - & 0.57 & Left-sided grade III sulcus \\
13. & 0.43 & - & Right-sided grade II sulcus \\
14. & 0.50 & - & Right-sided grade III sulcus \\
\hline
\end{tabular}

\section{DISCUSSION}

The classic findings in sulcus vocalis were noted by Hirano et $\mathrm{al}^{4}$ way back in 1990 . However, sulcus vocalis remains one of the most commonly missed glottic pathologies. As observed by Poels et al, ${ }^{9}$ Dailey and Zeitels ${ }^{10}$ noted that sulcus vocalis was the single most unidentified benign glottic entity. ${ }^{10}$ These undiagnosed sulcus vocalis are usually reported as 'phonatory gap' which is a sign and not a diagnosis.

The usual causes of a phonatory gap, without other structural or neuromuscular glottic pathologies, include sulcus vocalis, vocal fold atrophy and, less frequently, chronic laryngitis. ${ }^{11}$ Vocal fold atrophy (presbyphonia) more often leads to a symmetrical phonatory gap. ${ }^{12}$ Sulcus vocalis, on the contrary, causes an asymmetric or ' $\mathrm{D}$ ' shaped phonatory gap, as observed in our study.

Our study introduces a new parameter to help in diagnosing sulcus vocalis using rigid white light laryngoscopy, which is one of the most easily available diagnostic facilities. An angled rigid telescope with recording facility is available in most peripheral institutes. The use of white light laryngoscopy, as mentioned in this study, also lends objectivity to an otherwise subjective diagnosis. Prior studies have mentioned the use of digitized stroboscopes for determining the phonatory gap, which may not be widely available. ${ }^{13,14}$ As per our study, the gap can be measured with the help of a printout of a recorded white light image and a ruler. On analysis, if the phonatory gap is asymmetric, then the diagnosis of sulcus vocalis should be suspected, which should then be confirmed on stroboscopy. Hence, we suggest that asymmetric phonatory gaps on white light laryngoscopy be given the provisional diagnosis of 'suspected sulcus vocalis' and not 'phonatory gap.'

\section{CONCLUSION}

An asymmetric phonatory gap on white light laryngoscopy with no obvious structural (cyst, polyp, nodule, papilloma, contact granuloma, leukoplakia, suspected vocal fold malignancy) or neuromuscular (vocal fold paresis/palsy) glottic pathology should make the laryngologist suspect a sulcus vocalis. The final diagnosis, however, should be made with stroboscopy.

\section{REFERENCES}

1. Dailey SH, Ford CN. Surgical management of sulcus vocalis and vocal fold scarring. Otolaryngol Clin North Am 2006 Feb;39(1):23-42.

2. Ford CN, Inagi K, Khidr A, Bless DM, Gilchrist KW. Sulcus vocalis: a rational analytical approach to diagnosis and management. Ann Otol Rhinol Laryngol 1996 Mar;105(3): 189-200.

3. Benninger MS, Alessi D, Archer S, Bastian R, Ford C, Koufman J, Sataloff RT, Spiegel JR, Woo P. Vocal fold scarring: current concepts and management. Otolaryngol Head Neck Surg 1996 Nov;115(5):474-482.

4. Hirano M, Yoshida T, Tanaka S, Hibi S. Sulcus vocalis: functional aspects. Ann Otol Rhinol Laryngol 1990 Sep;99 (9 Pt 1):679-683.

5. Hansen JK, Thibeault SL. Current understanding and review of the literature: vocal fold scarring. J Voice 2006 Mar;20(1):110-120.

6. Itoh T, Kawasaki H, Morikawa I, Hirano M. Vocal fold furrows: a 10-year review of 240 patients. Auris Nasus Larynx 1983;10(Suppl):S17-S26.

7. Sunter AV, Yigit O, Hug GE, Alkan Z, Kocak I, Buyuk Y. Histopathological characteristics of sulcus vocalis. Otolaryngol Head Neck Surg 2011 Aug;145(2):264-269.

8. Byeon HK, Kim JH, Kwon JH, Jo KH, Hong HJ, Choi HS. Clinical characteristics of vocal polyps with underlying sulcus vocalis. J Voice 2013 Sep;27(5):632-635.

9. Poels PJ, de Jong FI, Schutte HK. Consistency of the preoperative and intraoperative diagnosis of benign vocal fold lesions. J Voice 2003 Sep;17(3):425-433.

10. Dailey SH, Spanou K, Zeitels SM. The evaluation of benign glottic lesions: rigid telescopic stroboscopy versus suspension microlaryngoscopy. J Voice 2007 Jan;21(1):112-118.

11. Lindestad PA, Hertegard S. Spindle-shaped glottal insufficiency with and without sulcus vocalis: a retrospective study. Ann Otol Rhinol Laryngol 1994 Jul;103(7):547-553.

12. Woo P, Casper J, Colton R, Brewer D. Dysphonia in the aging: physiology versus disease. Laryngoscope 1992 Feb;102(2): 139-144.

13. Omori K, Slavit DH, Kacker A, Blaugrund SM. Influence of size and etiology of glottal gap in glottic incompetence dysphonia. Laryngoscope 1998 Apr;108(4 Pt 1):514-518.

14. Omori K, Kacker A, Slavit DH, Blaugrund SM. Quantitative videostroboscopic measurement of glottal gap and vocal function: an analysis of thyroplasty type I. Ann Otol Rhinol Laryngol 1996 Apr;105(4):280-285. 